\title{
Asymptotic symmetries in Carrollian theories of gravity
}

\author{
Alfredo Pérez \\ Centro de Estudios Científicos (CECs), \\ Avenida Arturo Prat 514, Valdivia, Chile \\ E-mail: aperez@cecs.cl
}

ABstract: Asymptotic symmetries in Carrollian gravitational theories in $3+1$ space and time dimensions obtained from "magnetic" and "electric" ultrarelativistic contractions of General Relativity are analyzed. In both cases, parity conditions are needed to guarantee a finite symplectic term, in analogy with Einstein gravity. For the magnetic contraction, when Regge-Teitelboim parity conditions are imposed, the asymptotic symmetries are described by the Carroll group. With Henneaux-Troessaert parity conditions, the asymptotic symmetry algebra corresponds to a BMS-like extension of the Carroll algebra. For the electric contraction, because the lapse function does not appear in the boundary term needed to ensure a well-defined action principle, the asymptotic symmetry algebra is truncated, for Regge-Teitelboim parity conditions, to the semidirect sum of spatial rotations and spatial translations. Similarly, with Henneaux-Troessaert parity conditions, the asymptotic symmetries are given by the semidirect sum of spatial rotations and an infinite number of parity odd supertranslations. Thus, from the point of view of the asymptotic symmetries, the magnetic contraction can be seen as a smooth limit of General Relativity, in contrast to its electric counterpart.

KEywords: Classical Theories of Gravity, Space-Time Symmetries, Gauge-gravity correspondence

ArXiv EPrint: 2110.15834 


\section{Contents}

1 Introduction 2

2 Asymptotic symmetries in Magnetic Carrollian gravity 4

2.1 Action principle, variation of the charge and transformation laws 4

$\begin{array}{ll}2.2 & \text { Asymptotic behavior of the fields }\end{array}$

2.2.1 Fall-off of the canonical variables 6

$\begin{array}{ll}2.2 .2 \text { Variation of the charge } & 7\end{array}$

$\begin{array}{lll}2.2 .3 & \text { Transformation laws } & 8\end{array}$

2.2.4 Symplectic term $\quad 9$

2.3 Asymptotic symmetries with Regge-Teitelboim parity conditions 9

$\begin{array}{lll}2.3 .1 & \text { Parity conditions } & 9\end{array}$

$\begin{array}{ll}\text { 2.3.2 Charges and asymptotic symmetry algebra } & 10\end{array}$

2.4 Asymptotic symmetries with Henneaux-Troessaert parity conditions 11

$\begin{array}{lll}2.4 .1 & \text { Parity conditions } & 11\end{array}$

$\begin{array}{ll}\text { 2.4.2 Charges and asymptotic symmetry algebra } & 12\end{array}$

2.5 Example: Magnetic Carrollian Schwarzschild-like solution 13

3 Asymptotic symmetries in Electric Carrollian gravity $\quad 14$

3.1 Action principle, variation of the charge and transformation laws 14

$\begin{array}{lll}3.2 & \text { Asymptotic behavior of the fields } & 16\end{array}$

$\begin{array}{lll}3.2 .1 & \text { Fall-off of the canonical variables } & 16\end{array}$

$\begin{array}{ll}3.2 .2 \text { Variation of the charge } & 16\end{array}$

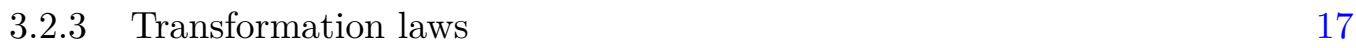

$\begin{array}{ll}3.2 .4 & \text { Symplectic term } \\ & 17\end{array}$

$\begin{array}{lll}3.3 & \text { Asymptotic symmetries with Regge-Teitelboim parity conditions } & 17\end{array}$

$\begin{array}{lll}\text { 3.3.1 Parity conditions } & 17\end{array}$

$\begin{array}{ll}\text { 3.3.2 Charges and asymptotic symmetry algebra } & 18\end{array}$

3.4 Asymptotic symmetries with Henneaux-Troessaert parity conditions 19

$\begin{array}{lll}3.4 .1 & \text { Parity conditions } & 19\end{array}$

$\begin{array}{ll}\text { 3.4.2 Charges and asymptotic symmetry algebra } & 19\end{array}$

3.5 Example: Electric Carrollian Schwarzschild-like solution 20

4 Concluding remarks $\quad 21$

A Analysis in Cartesian coordinates with Regge-Teitelboim parity conditions

A.1 Magnetic Carrollian gravity 24

$\begin{array}{lll}\text { A.2 } & \text { Electric Carrollian gravity } 24\end{array}$ 


\section{Introduction}

The Carroll symmetry was introduced by Levy-Leblond in 1965 as an "ultrarelativistic" limit of the Poincare symmetry, i.e., the limit in which the speed of light vanishes $(c \rightarrow$ 0 ) [1]. The Carroll algebra belongs to the family of Lie algebras associated with the possible "kinematical groups" classified in ref. [2], and has been used in very different physical contexts (see e.g. [3-35] and references therein). In this ultrarelativistic limit, light cones close up, and as a consequence neighboring points become causally disconnected. In the words of Levy-Leblond:

"In a world whose invariance group would be this new group, there would be practically no causality."

It has recently been shown that relativistic field theories generically admit two inequivalent contractions that lead to Carroll invariant field theories [36]. They were termed "electric" and "magnetic" contractions, respectively. They were known in the case of electrodynamics [6], but remarkably they also exist in theories that are not necessarily invariant under duality transformations. Both contractions, in the case of scalar and electromagnetic fields, were also discussed in ref. [37].

In the context of gravitation, the theory obtained from the electric contraction of General Relativity has been known for a long time, and can be interpreted as a strong coupling limit of Einstein gravity [38], or as a gravitational theory in a "zero-signature limit" [39]. In ref. [40] it was written in terms of a covariant Lagrangian under Carroll transformations, and in refs. [41-43] it was used as a starting point of an alternative perturbative approach in terms of the signature parameter for the quantization of the gravitational field, mimicking the quantization process of a relativistic free particle. By virtue of their "ultra-local" properties, this theory can also be used in the description of the spacetime near space-like singularities [44-47]. On the other hand, the magnetic contraction was recently introduced by Henneaux and Salgado-Rebolledo in ref. [36], and is described in Hamiltonian form. ${ }^{1}$

Both contractions share a common characteristic: the algebra of first class constraints possesses a Carroll structure. In other words, the normal surface deformations form an abelian subgroup, in sharp contrast with the surface deformation algebra of General Relativity $[50,51]$. However, the fact that the algebra of constraints has a Carroll structure in the sense described above, is not enough to guarantee that the theory is in fact Carrollian. The theory must have a Carroll symmetry (or an extension of it) emerging as an asymptotic symmetry defined by appropriate improper (large) gauge transformations that change the physical state [52]. Therefore, it is of fundamental importance to study the asymptotic structure of these theories, and to determine which of them possess Carrollian asymptotic symmetries with well-defined canonical generators. This is the main purpose of this article.

\footnotetext{
${ }^{1}$ Different actions for gravitational theories with Carrollian symmetries were constructed in refs. [48, 49] by gauging the Carroll algebra. The relation with the electric and magnetic contractions discussed in ref. [36] is still unclear. However, in the same reference it was conjectured that the electric contraction could be related with the action in [48], while the magnetic contraction to the one introduced in [49].
} 
Action principle in Hamiltonian form

$$
\begin{gathered}
I=\int d t d^{3} x\left(\pi^{i j} \dot{g}_{i j}-N \mathcal{H}\right. \\
\left.-N^{i} \mathcal{H}_{i}\right)
\end{gathered}
$$

$$
I=\int d t d^{3} x\left(\pi^{i j} \dot{g}_{i j}-N \mathcal{H}^{M}\right.
$$$$
I=\int d t d^{3} x\left(\pi^{i j} \dot{g}_{i j}-N \mathcal{H}^{E}\right.
$$$$
\mathcal{H}=\frac{1}{\sqrt{g}}\left(\pi^{i j} \pi_{i j}-\frac{1}{2} \pi^{2}\right)-\sqrt{g} R
$$$$
\mathcal{H}^{M}=-\sqrt{g} R
$$$$
\mathcal{H}^{E}=\frac{1}{\sqrt{g}}\left(\pi^{i j} \pi_{i j}-\frac{1}{2} \pi^{2}\right)
$$

$$
\mathcal{H}_{i}=-2 \pi_{i \mid j}^{j}
$$$$
\mathcal{H}_{i}^{M}=-2 \pi_{i \mid j}^{j}
$$$$
\mathcal{H}_{i}^{E}=-2 \pi_{i \mid j}^{j}
$$

\footnotetext{
Poisson bracket between two

Hamiltonian constraints$$
\text { constraints }
$$

$$
\left\{\mathcal{H}^{E}(x), \mathcal{H}^{E}\left(x^{\prime}\right)\right\}=0
$$$$
\left.+g^{i j}\left(x^{\prime}\right) \mathcal{H}_{j}\left(x^{\prime}\right)\right) \delta,_{i}\left(x, x^{\prime}\right)
$$

$$
\left\{\mathcal{H}^{M}(x), \mathcal{H}^{M}\left(x^{\prime}\right)\right\}=0
$$$$
\left.-N^{i} \mathcal{H}_{i}^{E}\right)
$$

$$
\begin{array}{cc}
\delta H=\oint d^{2} s_{l}\left[2 N_{k} \delta \pi^{k l}\right. & \delta H_{M}=\oint d^{2} s_{l}\left[2 N_{k} \delta \pi^{k l}\right. \\
+\left(2 N^{k} \pi^{j l}-N^{l} \pi^{j k}\right) \delta g_{j k} & +\left(2 N^{k} \pi^{j l}-N^{l} \pi^{j k}\right) \delta g_{j k} \\
\left.+G^{i j k l}\left(N \delta g_{i j \mid k}-N_{\mid k} \delta g_{i j}\right)\right] & \left.+G^{i j k l}\left(N \delta g_{i j \mid k}-N_{\mid k} \delta g_{i j}\right)\right]
\end{array}
$$

$\delta H_{E}=\oint d^{2} s_{l}\left[2 N_{k} \delta \pi^{k l}\right.$ $\left.+\left(2 N^{k} \pi^{j l}-N^{l} \pi^{j k}\right) \delta g_{j k}\right]$ There is no $N$ (lapse) in the expression

Need for parity conditions?

Asymptotic symmetry algebra

with Regge-

Teitelboim parity conditions

Asymptotic symmetry algebra with Henneaux -Troessaert parity conditions Poincaré algebra

Carroll algebra

Yes

Yes

Yes

(in an unconventional

basis)
BMS-like extension of the Carroll algebra

Spatial rotations $₫$ parity odd supertranslations

(spatial translations obtained from the modes with $\ell=1$ in the spherical harmonics expansion)

Table 1. General Relativity and Electric/Magnetic Carrollian gravity compared and contrasted. 
As in the case of General Relativity, it will be necessary to introduce suitable parity conditions to have a finite symplectic term. Two possibilities are explored, analogous to Regge-Teitelboim [53] and Henneaux-Troessaert [54] parity conditions at spatial infinity. Thus, the asymptotic symmetry algebra will depend on the type of contraction, electric or magnetic, as well as on the choice of parity conditions. In particular, for the magnetic contraction, the asymptotic symmetries are described by the finite-dimensional Carroll group when Regge-Teitelboim parity conditions are imposed. With Henneaux-Troessaert parity conditions, the asymptotic symmetry algebra corresponds to a BMS-like extension of the Carroll algebra. On the other hand, in the case of the electric contraction, and because the lapse function does not appear in the boundary term needed to ensure a well-defined action principle, the asymptotic symmetry algebra is truncated to the semi-direct sum of spatial rotations and spatial translations when Regge-Teitelboim parity conditions are used. Similarly, with Henneaux-Troessaert parity conditions, the asymptotic symmetries are given by the semi-direct sum of spatial rotations and an infinite number of parity odd supertranslations. Hence, the Carroll group is not present in the electric contraction, and consequently, there is no generator associated with time translations that allow defining energy in this theory. In this sense, from the point of view of the asymptotic symmetries, the magnetic contraction can be seen as a smooth limit of General Relativity, in contrast to its electric counterpart. The results are summarized and compared with the standard ones in General Relativity in table 1.

The plan of the paper is the following: in the next section, the asymptotic symmetries of the theory obtained from the magnetic Carrollian contraction of General Relativity are studied. In particular, we consider the cases when Regge-Teitelboim parity conditions and Henneaux-Troessaert parity conditions are implemented. The asymptotic symmetry algebra is then determined, and the canonical realization of the charges is obtained for each case. As an example, the charges associated with a "Carrollian Schwarzschild-like" configuration are computed explicitly. In section 3, a similar analysis is performed for the case of the "Electric Carrollian gravity." Both, Regge-Teitelboim and Henneaux-Troessaert parity conditions are considered, and the asymptotic symmetry algebra together with their canonical generators are determined for each case. The charges associated with a "Carrollian Schwarzschild-like" solution of the theory are also computed. Section 4 is devoted to conclusions and final remarks. Finally, in appendix A, the results obtained in section 2 and section 3 for the case of Regge-Teitelboim parity conditions are reviewed in Cartesian coordinates.

\section{Asymptotic symmetries in Magnetic Carrollian gravity}

\subsection{Action principle, variation of the charge and transformation laws}

The Magnetic Carrollian theory of gravity was recently introduced in ref. [36]. Its action principle is obtained from the action of General Relativity in Hamiltonian form by the socalled "magnetic contraction," and is described in the canonical formalism. The canonical 
action is given by

$$
I=\int d t d^{3} x\left(\pi^{i j} \dot{g}_{i j}-N \mathcal{H}^{M}-N^{i} \mathcal{H}_{i}^{M}\right)
$$

where

$$
\mathcal{H}^{M}=-\sqrt{g} R, \quad \mathcal{H}_{i}^{M}=-2 \pi_{i \mid j}^{j} .
$$

The canonical variables are given by $g_{i j}$ and $\pi^{i j}$, with $i, j=1,2,3$. Here $g_{i j}$ are the spatial metric components of the corresponding four-dimensional Carrollian metric [40], and $\pi^{i j}$ denotes their conjugate momenta. In analogy with General Relativity, the functions $N$ and $N^{i}$ will be called "lapse" and "shift" functions, respectively. These are the Lagrange multipliers that implement the constraints $\mathcal{H}^{M} \approx 0$ and $\mathcal{H}_{i}^{M} \approx 0$ defined in eq. (2.2), where $R$ is the Ricci scalar of the three-dimensional metric $g_{i j}$, and | denotes covariant differentiation with respect to this metric.

The first class constraints obey the following surface deformation algebra

$$
\begin{aligned}
& \left\{\mathcal{H}^{M}(x), \mathcal{H}^{M}\left(x^{\prime}\right)\right\}=0 \\
& \left\{\mathcal{H}^{M}(x), \mathcal{H}_{i}^{M}\left(x^{\prime}\right)\right\}=\mathcal{H}^{M}(x) \delta_{, i}\left(x, x^{\prime}\right) \\
& \left\{\mathcal{H}_{i}^{M}(x), \mathcal{H}_{j}^{M}\left(x^{\prime}\right)\right\}=\mathcal{H}_{i}^{M}\left(x^{\prime}\right) \delta_{, j}\left(x, x^{\prime}\right)+\mathcal{H}_{j}^{M}(x) \delta_{, i}\left(x, x^{\prime}\right) .
\end{aligned}
$$

Note that the Poisson bracket between two $\mathcal{H}^{M}$ in eq. (2.3) vanishes, while the Poisson brackets in eqs. (2.4) and (2.5) coincide with those of General Relativity [50]. Indeed, the above algebra can be obtained from the "zero signature limit" of the surface deformation algebra of Einstein gravity when the parameter defining the signature is set to zero, i.e., when $\epsilon=0$ in eqs. (28.a)-(28.c) of ref. [50]. Furthermore, as it was pointed out in [39], the commutations relations (2.3)-(2.5) define a true algebra in the sense that the structure constants are independent of the fields, in contrast to General Relativity (see table 1). The abelian subalgebra spanned by $\mathcal{H}^{M}(x)$ is characteristic of the Carroll symmetry because the generators that are associated with normal deformations, i.e., the generators of time translations and boosts, commute among them.

Hamilton equations follow directly from the action principle (2.1). They read

$$
\begin{aligned}
& \dot{g}_{i j}=N_{i \mid j}+N_{j \mid i}, \\
& \dot{\pi}^{i j}=-N \sqrt{g}\left(R^{i j}-\frac{1}{2} g^{i j} R\right)+\sqrt{g}\left(N^{|i| j}-g^{i j} N_{\mid k}^{\mid k}\right)+\left(N^{k} \pi^{i j}\right)_{\mid k}-N_{\mid k}^{i} \pi^{k j}-N_{\mid k}^{j} \pi^{k i} .
\end{aligned}
$$

The momenta cannot be eliminated in terms of time derivatives of $g_{i j}$, as it can be directly seen from eq. (2.6). This is because $\mathcal{H}^{M}$ is independent of the momenta, while $\mathcal{H}_{i}^{M}$ is linear in them.

The generator of gauge symmetries takes the form

$$
G\left[\xi, \xi^{i}\right]=\int d^{3} x\left(\xi \mathcal{H}^{M}+\xi^{i} \mathcal{H}_{i}^{M}\right)+Q_{M}
$$

where, according to Regge and Teitelboim, $Q_{M}$ is the boundary term that must be added in order to guarantee that the canonical generators have well-defined functional derivatives [53]. Its variation then reads

$$
\delta Q_{M}=\oint d^{2} s_{l}\left[G^{i j k l}\left(\xi \delta g_{i j \mid k}-\xi_{\mid k} \delta g_{i j}\right)+2 \xi_{k} \delta \pi^{k l}+\left(2 \xi^{k} \pi^{j l}-\xi^{l} \pi^{j k}\right) \delta g_{j k}\right],
$$


where $G^{i j k l}$ is the inverse of the de Witt supermetric, given by

$$
G^{i j k l}=\frac{1}{2} \sqrt{g}\left(g^{i k} g^{j l}+g^{i l} g^{j k}-2 g^{i j} g^{k l}\right) .
$$

Note that the expression for the variation of the charge (2.9) precisely coincides with the one in General Relativity. The reason is that the term that was removed from $\mathcal{H}$ in the process of contraction, $G_{i j k l} \pi^{i j} \pi^{k l}$, does not contain spatial derivatives, and therefore it does not contribute to the boundary term.

The transformation laws for the canonical variables $g_{i j}$ and $\pi^{i j}$ are then generated by (2.8). They are given by

$$
\begin{aligned}
\delta g_{i j} & =\xi_{i \mid j}+\xi_{j \mid i} \\
\delta \pi^{i j} & =-\xi \sqrt{g}\left(R^{i j}-\frac{1}{2} g^{i j} R\right)+\sqrt{g}\left(\xi^{|i| j}-g^{i j} \xi_{\mid k}^{\mid k}\right)+\left(\xi^{k} \pi^{i j}\right)_{\mid k}-\xi_{\mid k}^{i} \pi^{k j}-\xi_{\mid k}^{j} \pi^{k i} .
\end{aligned}
$$

The transformation laws take exactly the same form as the Hamilton equations in (2.6), (2.7) with the replacement $\xi \rightarrow N, \xi^{i} \rightarrow N^{i}$. This is due to the fact that the time evolution is a gauge transformation.

\subsection{Asymptotic behavior of the fields}

For the analysis of the asymptotic symmetries it is necessary to specify the behavior of the fields, in this case the canonical variables $g_{i j}$ and $\pi^{i j}$, in the asymptotic region. Then one has to find the most general form of the parameters $\xi, \xi^{i}$ that preserves the fall-off of the canonical variables under the set of transformations defined in eqs. (2.10) and (2.11). The transformations with associated non-trivial charges will then define the asymptotic symmetries of the theory. In addition, the asymptotic behavior of the fields must guarantee that the charges are finite and integrable in the functional sense, and that the symplectic term is finite.

\subsubsection{Fall-off of the canonical variables}

To describe the asymptotic behavior of the fields, we will consider deviations with respect to the "Carrollian background configuration" characterized by the canonical variables $\bar{g}_{i j}$, $\bar{\pi}^{i j}$ given by

$$
\bar{g}_{i j} d x^{i} d x^{j}=d r^{2}+r^{2} \gamma_{A B} d x^{A} d x^{B}, \quad \bar{\pi}^{i j}=0,
$$

with $A, B=1,2$, and where $\gamma_{A B}$ denotes the metric of the round 2 -sphere. Since $\bar{g}_{i j}$ corresponds to the metric of the three-dimensional Euclidean flat space, this configuration automatically solves the constraints in eq. (2.2).

In what follows, we will use spherical coordinates because they will allow us to discuss the parity conditions of Regge-Teitelboim [53] and Henneaux-Troessaert [54] using the same expressions. The analysis in Cartesian coordinates for the case of Regge-Teitelboim parity conditions is discussed in appendix A. 
The proposed fall-off for the canonical variables is given by

$$
\begin{aligned}
g_{r r} & =1+\frac{f_{r r}}{r}+\frac{f_{r r}^{(-2)}}{r^{2}}+\mathcal{O}\left(r^{-3}\right), \\
g_{r A} & =\frac{f_{r A}^{(-1)}}{r}+\mathcal{O}\left(r^{-2}\right), \\
g_{A B} & =r^{2} \gamma_{A B}+r f_{A B}+f_{A B}^{(0)}+\mathcal{O}\left(r^{-1}\right), \\
\pi^{r r} & =p^{r r}+\mathcal{O}\left(r^{-1}\right) \\
\pi^{r A} & =\frac{p^{r A}}{r}+\frac{p_{(-2)}^{r A}}{r^{2}}+\mathcal{O}\left(r^{-3}\right), \\
\pi^{A B} & =\frac{p^{A B}}{r^{2}}+\mathcal{O}\left(r^{-3}\right) .
\end{aligned}
$$

This is exactly the same asymptotic behavior for the fields used in the case of General Relativity $[53,54]$ and guarantees that the charges are finite and integrable in the functional sense. The $\mathcal{O}(1)$ term of $g_{r A}$ has been set zero as in ref. [54] to avoid difficulties with the integrability. Note that all the terms that are relevant for the charges have been explicitly displayed.

The asymptotic conditions (2.13)-(2.18) are preserved under the transformations given in eqs. (2.10) and (2.11) with gauge parameters of the form

$$
\begin{aligned}
\xi & =r b+f(\theta, \phi)+\mathcal{O}\left(r^{-1}\right), \\
\xi^{r} & =W(\theta, \phi)+\mathcal{O}\left(r^{-1}\right), \\
\xi^{A} & =Y^{A}+\frac{\partial^{A} W(\theta, \phi)}{r}+\mathcal{O}\left(r^{-2}\right),
\end{aligned}
$$

with

$$
b=\vec{\beta} \cdot \hat{r}, \quad Y^{A}=\frac{\epsilon^{A B}}{\sqrt{\gamma}} \partial_{B}(\vec{\omega} \cdot \hat{r}) .
$$

Here, $\epsilon^{A B}$ is the antisymmetric Levi-Civita symbol and $\gamma$ denotes the determinant of the 2-sphere metric $\gamma_{A B}$. The indices $A, B$ are lowered and raised with the same metric. The vector $\hat{r}=(\sin \theta \cos \phi, \sin \theta \sin \phi, \cos \theta)$ is the unit normal to the 2 -sphere, and the threedimensional constant vectors $\vec{\beta}$ and $\vec{\omega}$ are the parameters associated with boosts and spatial rotations, respectively. The functions $f=f(\theta, \phi)$ and $W=W(\theta, \phi)$ are general arbitrary scalar functions defined on the 2 -sphere.

\subsubsection{Variation of the charge}

The variation of the charge is obtained by replacing the asymptotic conditions (2.13)-(2.18) and the asymptotic form of the gauge parameters (2.19)-(2.22) in the expression (2.9) for $\delta Q_{M}$. It contains a divergent term in the large $r$ expansion that can be eliminated by using the order $\mathcal{O}\left(r^{-1}\right)$ of the constraint $\mathcal{H}^{M}$ given by

$$
\Delta f_{r r}+\Delta \tilde{f}-D^{A} D^{B} f_{A B}=0,
$$


and the order $\mathcal{O}(1)$ of the constraint $\mathcal{H}_{A}^{M}$

$$
p^{r A}+D_{B} p^{A B}=0,
$$

as it was done by Henneaux and Troessaert in the case of General Relativity [54]. Here $D_{A}$ denotes the covariant derivative with respect to the metric of the 2 -sphere $\gamma_{A B}$, and $\Delta=D_{A} D^{A}$ is the corresponding Laplacian. We are using the notation $\tilde{X}:=\gamma^{A B} X_{A B}$ to represent the trace of a tensor $X_{A B}$.

The divergent part of the charge reads

$$
Q_{M}^{\mathrm{div}}=r \oint d^{2} x 2 \sqrt{\gamma}\left[(\vec{\beta} \cdot \hat{r})\left(f_{r r}+\frac{1}{2} \tilde{f}\right)+p^{r A} \epsilon_{A C} D^{C}(\vec{\omega} \cdot \hat{r})\right] .
$$

Using the constraints (2.23), (2.24) and performing appropriate integrations by parts, one can show that the divergent term vanishes identically

$$
Q_{M}^{\mathrm{div}}=0 .
$$

The finite part of the variation of the charge is given by

$$
\begin{aligned}
\delta Q_{M}=\delta \oint d^{2} x\left[f\left(2 \sqrt{\gamma} f_{r r}\right)+2 Y^{A}\left(p_{A(-2)}^{r}+f_{A B} p^{r B}\right)+2 W\left(p^{r r}-D_{A} p^{r A}\right)\right. \\
\left.+\sqrt{\gamma} b\left(2 f_{r r}^{(-2)}+2 D^{A} f_{r A}^{(-1)}+2 \tilde{f}^{(0)}-\frac{3}{2} f_{r r}^{2}-\frac{3}{4} f^{A B} f_{A B}+\frac{\tilde{f}^{2}}{4}\right)\right] \\
+\oint d^{2} x \frac{\sqrt{\gamma} b}{2}\left(\tilde{f} \delta f_{r r}-f_{r r} \delta \tilde{f}\right) .
\end{aligned}
$$

This expression contains a non-integrable part that will be eliminated by the parity conditions.

\subsubsection{Transformation laws}

The transformation laws of the fields that are relevant for the discussion of the parity conditions are the following:

$$
\begin{aligned}
\delta f_{r r}= & \mathcal{L}_{Y} f_{r r} \\
\delta f_{A B}= & \mathcal{L}_{Y} f_{A B}+2\left(D_{A} D_{B}+\gamma_{A B}\right) W \\
\delta p^{r r}= & \mathcal{L}_{Y} p^{r r}+b \frac{\sqrt{\gamma}}{2}\left[6 f_{r r}+D^{A} D^{B} f_{A B}-\tilde{f}-\Delta \tilde{f}\right]+\sqrt{\gamma}\left(D^{A} b\right)\left(D^{B} f_{A B}\right) \\
& -\frac{\sqrt{\gamma}}{2}\left(D^{A} b\right)\left(D_{A} \tilde{f}\right)-\sqrt{\gamma} \Delta f \\
\delta p^{r A}= & \mathcal{L}_{Y} p^{r A}+\frac{\sqrt{\gamma}}{2} D_{B}\left(b f^{A B}\right)-\frac{\sqrt{\gamma}}{2} b D^{A}\left(\tilde{f}+2 f_{r r}\right)-\sqrt{\gamma} D^{A} f \\
\delta p^{A B}= & \mathcal{L}_{Y} p^{A B}+\frac{\sqrt{\gamma}}{2} b\left(D^{A} D^{B} f_{r r}-\gamma^{A B} \Delta f_{r r}+f^{A B}\right) \\
& +\frac{\sqrt{\gamma}}{2}\left[\left(D^{A} b\right)\left(D_{C} f^{C B}\right)+\left(D^{B} b\right)\left(D_{C} f^{C A}\right)\right] \\
& -\frac{\sqrt{\gamma}}{2}\left[\left(D^{A} b\right)\left(D^{B} \tilde{f}\right)+\left(D^{B} b\right)\left(D^{A} \tilde{f}\right)\right]-\frac{\sqrt{\gamma}}{2}\left(D^{C} b\right)\left(D_{C} f^{A B}\right) \\
& +\frac{\sqrt{\gamma}}{2} \gamma^{A B}\left(D^{C} b\right) D_{C}\left(\tilde{f}-f_{r r}\right)+\sqrt{\gamma}\left(D^{A} D^{B}-\gamma^{A B} \Delta\right) f .
\end{aligned}
$$


Here, $\mathcal{L}_{Y}$ denotes the Lie derivative with respect to the vector field on the sphere $Y^{A}$. Note that the field $f_{r r}$, which in the variation of the charge (2.25) is associated with the term of order $\mathcal{O}(1)$ in the time translations, transforms only under spatial rotations in contrast to the case of General Relativity. This is the imprint, at the level of the transformation laws of the fields, of the Carrollian structure of the theory.

\subsubsection{Symplectic term}

For large values of $r$, the symplectic term takes the form

$$
\int d t d^{3} x \pi^{i j} \dot{g}_{i j} \underset{r \rightarrow \infty}{=} \log (r) \int d t \oint d^{2} x\left(p^{r r} \dot{f}_{r r}+p^{A B} \dot{f}_{A B}\right)+\mathcal{O}\left(r^{-1}\right) .
$$

It possesses a logarithmic divergence that can be removed by imposing appropriate parity conditions on the canonical variables. Since the symplectic term is identical to the one in General Relativity, one can use the same Regge-Teitelboim [53] and HenneauxTroessaert [54] parity conditions that were used in Einstein gravity.

\subsection{Asymptotic symmetries with Regge-Teitelboim parity conditions}

\subsubsection{Parity conditions}

Regge-Teitelboim parity conditions were introduced in ref. [53] in the context of the study of asymptotically flat spacetimes in General Relativity. In that case, they cancel the logarithmic divergence in the symplectic term, and the asymptotic symmetry algebra reduces to the Poincaré algebra. In this section, the same Regge-Teitelboim parity condition will be used to remove the divergence in the symplectic term (2.31) of the magnetic Carrollian theory.

In spherical coordinates, the antipodal map on the 2-sphere is obtained from the following transformation

$$
\begin{aligned}
& \theta \rightarrow-\theta+\pi, \\
& \phi \rightarrow \phi+\pi .
\end{aligned}
$$

The Regge-Teitelboim parity condition in spherical coordinates are then given by

$$
\begin{aligned}
f_{i j} d x^{i} d x^{j} & \text { (even under the antipodal map) } \\
p^{i j} \partial_{i} \partial_{j} & \text { (odd under the antipodal map) }
\end{aligned}
$$

Explicitly, they read

$$
\begin{aligned}
& f_{r r}, f_{\theta \theta}, f_{\phi \phi}, p^{\theta \phi}, p^{r \theta} \quad \text { (parity even) } \\
& f_{\theta \phi}, p^{r r}, p^{\theta \theta}, p^{\phi \phi}, p^{r \phi} \quad \text { (parity odd) }
\end{aligned}
$$

The logarithmic divergence in the symplectic term in eq. (2.31) only contains fields of opposite parity. Therefore, it vanishes after the integration on the 2 -sphere.

For consistency, the parity conditions (2.32), (2.33) must be preserved under the transformation laws of the fields, imposing additional restrictions on the gauge parameters. 
From the transformation of $f_{A B}$ in eq. (2.27), $W$ must be even, with the exception of the mode that satisfies $\left(D_{A} D_{B}+\gamma_{A B}\right) W=0$. From the trace of this equation, it becomes clear that the mode with $\ell=1$ in the expansion in spherical harmonics obeys this restriction. Thus, one has

$$
W=\vec{\alpha} \cdot \hat{r}+W_{\text {even }}(\theta, \phi)
$$

Here, $\vec{\alpha}$ is a constant vector field that will be associated with spatial translations, and $W_{\text {even }}$ contains only spherical harmonics of even parity, i.e., those with even values of $\ell$.

From the transformation law of $p^{r r}$ in eq. (2.28), $f$ must be parity odd, with the exception of the mode that satisfies $\Delta f=0$, i.e., the mode with $\ell=0$. Therefore,

$$
f=T+f_{\text {odd }}(\theta, \phi)
$$

where $T$ is a constant, and $f_{\text {odd }}(\theta, \phi)$ contains parity odd spherical harmonics. With these restrictions on the parameters, all the transformation laws preserve the Regge-Teitelboim parity conditions.

\subsubsection{Charges and asymptotic symmetry algebra}

When the parity conditions (2.32) and (2.33) are implemented in the variation of the charge (2.25), all the quadratic terms vanish and the charge becomes integrable in the functional sense. Furthermore, there are no contributions to the charge coming from the parameters $W_{\text {even }}(\theta, \phi)$ and $f_{\text {odd }}(\theta, \phi)$, and therefore, they define transformations that are pure gauge (proper gauge transformations). The expression for the charge then simplifies to

$$
\begin{aligned}
Q_{M}=\oint d^{2} x 2 \sqrt{\gamma}[ & T f_{r r}+(\vec{\omega} \cdot \hat{r}) \epsilon_{A B} D^{A} p_{(-2)}^{r B}+\frac{(\vec{\alpha} \cdot \hat{r})}{\sqrt{\gamma}}\left(p^{r r}-D_{A} p^{r A}\right) \\
& \left.+(\vec{\beta} \cdot \hat{r})\left(f_{r r}^{(-2)}+D^{A} f_{r A}^{(-1)}+\tilde{f}^{(0)}\right)\right] .
\end{aligned}
$$

The asymptotic form of the gauge parameters expressed in terms of the parameters associated to improper (large) gauge transformations is then given by

$$
\begin{aligned}
\xi & =r(\vec{\beta} \cdot \hat{r})+T+\mathcal{O}\left(r^{-1}\right), \\
\xi^{r} & =\vec{\alpha} \cdot \hat{r}+\mathcal{O}\left(r^{-1}\right), \\
\xi^{A} & =\frac{\epsilon^{A B}}{\sqrt{\gamma}} \partial_{B}(\vec{\omega} \cdot \hat{r})+\frac{\partial^{A}(\vec{\alpha} \cdot \hat{r})}{r}+\mathcal{O}\left(r^{-2}\right) .
\end{aligned}
$$

The asymptotic symmetry algebra can then be obtained using the "Carrollian surface deformation algebra" (2.3)-(2.5). The commutator of two deformations with parameters $\left(\xi_{1}, \xi_{1}^{i}\right)$ and $\left(\xi_{2}, \xi_{2}^{i}\right)$ gives new parameters $\left(\xi_{3}, \xi_{3}^{i}\right)$ that are given by

$$
\begin{aligned}
& \xi_{3}=\xi_{1}^{i} \partial_{i} \xi_{2}-\xi_{2}^{i} \partial_{i} \xi_{1}, \\
& \xi_{3}^{i}=\xi_{1}^{j} \partial_{j} \xi_{2}^{i}-\xi_{2}^{j} \partial_{j} \xi_{1}^{i} .
\end{aligned}
$$

These expressions can be directly obtained by setting $\epsilon=0$ (zero signature limit) in eqs. (25.a) and (25.b) in ref. [50]. 
From the asymptotic form of the parameters (2.19)-(2.22), together with the expressions $W=\vec{\alpha} \cdot \hat{r}$ and $f=T$ coming from the parity conditions, we find

$$
\begin{array}{ll}
T_{3}=\delta_{I J}\left(\alpha_{1}^{I} \beta_{2}^{J}-\alpha_{2}^{I} \beta_{1}^{J}\right), & \alpha_{3}^{K}=-\epsilon_{I J K}\left(\alpha_{1}^{I} \omega_{2}^{J}-\alpha_{2}^{I} \omega_{1}^{J}\right), \\
\beta_{3}^{K}=-\epsilon_{I J K}\left(\beta_{1}^{I} \omega_{2}^{J}-\beta_{2}^{I} \omega_{1}^{J}\right), & \omega_{3}^{K}=-\epsilon_{I J K} \omega_{1}^{I} \omega_{2}^{J},
\end{array}
$$

where the indices $I, J, K=1,2,3$ label the components of the three-dimensional vectors $\vec{\alpha}, \vec{\beta}$ and $\vec{\omega}$. The above composition rule is precisely the one of the Carroll algebra $[1,2]$. Indeed, if

$$
\begin{aligned}
E & =2 \oint d^{2} x \sqrt{\gamma} f_{r r}, & P_{I} & =2 \oint d^{2} x \hat{r}_{I}\left(p^{r r}-D_{A} p^{r A}\right), \\
K_{I} & =2 \oint d^{2} x \sqrt{\gamma} \hat{r}_{I}\left(f_{r r}^{(-2)}+D^{A} f_{r A}^{(-1)}+\tilde{f}^{(0)}\right), & J_{I} & =2 \sqrt{\gamma} \oint d^{2} x \hat{r}_{I} \epsilon_{A B} D^{A} p_{(-2)}^{r B},
\end{aligned}
$$

the charge (2.34) takes the form

$$
Q_{M}=T E+\vec{\omega} \cdot \vec{J}+\vec{\alpha} \cdot \vec{P}+\vec{\beta} \cdot \vec{K}
$$

and the generators $E, P_{I}, K_{I}$ and $J_{I}$ fulfill the Carroll algebra with the following nonvanishing Poisson brackets

$$
\begin{aligned}
\left\{P_{I}, K_{J}\right\} & =\delta_{I J} E, & & \left\{J_{I}, J_{J}\right\}=-\epsilon_{I J K} J_{K}, \\
\left\{P_{I}, J_{J}\right\} & =-\epsilon_{I J K} P_{K}, & \left\{K_{I}, J_{I}\right\} & =-\epsilon_{I J K} K_{K} .
\end{aligned}
$$

Thus, the asymptotic symmetry algebra of the magnetic Carrollian theory of gravity with Regge-Teitelboim parity conditions is precisely the four-dimensional Carroll algebra of Levy-Leblond.

\subsection{Asymptotic symmetries with Henneaux-Troessaert parity conditions}

\subsubsection{Parity conditions}

In the case of General Relativity, a different set of parity conditions was recently introduced by Henneaux and Troessaert [54] that extends the asymptotic symmetry algebra at spatial infinity from the Poincaré algebra to the $\mathrm{BMS}_{4}$ algebra $[55,56]$. As it was discussed in section 2.2.4, since the symplectic term of the magnetic Carrollian theory takes exactly the same form as the one in Einstein gravity, the Henneaux-Troessaert parity conditions can also be implemented in the case of the Magnetic Carrollian gravity. The asymptotic symmetry algebra will then be enlarged from the Carroll algebra to a BMS-like extension of it, that includes additional "Carrollian supertranslations."

Following ref. [54], in order to implement the Henneaux-Troessaert parity conditions it is useful to introduce the following new variables

$$
\begin{aligned}
\bar{\lambda} & =\frac{1}{2} f_{r r}, & \bar{k}_{A B} & =\frac{1}{2} f_{A B}+\frac{1}{2} f_{r r} \gamma_{A B}, \\
\bar{p} & =2\left(p^{r r}-p^{A B} \gamma_{A B}\right), & k^{(2)} & =-\frac{f_{r r} \tilde{f}}{4}-\frac{3}{4} f_{r r}^{2}+f_{r r}^{(-2)}+D^{A} f_{r A}^{(-1)}+\tilde{f}_{(0)}-\frac{1}{2} f^{A B} f_{A B} .
\end{aligned}
$$


The Henneaux-Troessaert parity conditions are then defined by

$$
\begin{array}{cc}
\bar{\lambda}, p^{r \phi}, p^{\theta \theta}, p^{\phi \phi}, \bar{k}_{\theta \phi} & \text { (parity even) }, \\
\bar{p}, p^{r \theta}, p^{\theta \phi}, \bar{k}_{\theta \theta}, \bar{k}_{\phi \phi} & \text { (parity odd) } .
\end{array}
$$

In terms of these new variables the symplectic term (2.31) takes the form

$$
\int d t d^{3} x \pi^{i j} \dot{g}_{i j} \underset{r \rightarrow \infty}{=} \log (r) \int d t \oint d^{2} x\left(\overline{\bar{p}} \dot{\bar{\lambda}}+2 p^{A B} \dot{\bar{k}}_{A B}\right)+\mathcal{O}\left(r^{-1}\right)
$$

expression that vanishes by virtue of the parity conditions (2.41), (2.42). Therefore, the symplectic term is finite once the Henneaux-Troessaert parity conditions are imposed.

The parameters take the same form as in eqs. (2.19)-(2.22), with

$$
f=-\frac{1}{2} b\left(3 f_{r r}+\tilde{f}\right)+T(\theta, \phi)
$$

where the functions $T(\theta, \phi)$ and $W(\theta, \phi)$ have the following parity under the antipodal map

$$
\begin{aligned}
T(\theta, \phi) & \text { (parity even), } \\
W(\theta, \phi) & \text { (parity odd) } .
\end{aligned}
$$

The parity conditions (2.41), (2.42) are then preserved by the transformation laws of the fields, which in terms of the variables introduced in eqs. (2.40) read

$$
\begin{aligned}
\delta \bar{\lambda}= & \mathcal{L}_{Y} \bar{\lambda} \\
\delta \bar{k}_{A B}= & \mathcal{L}_{Y} \bar{k}_{A B}+\left(D_{A} D_{B}+\gamma_{A B}\right) W \\
\delta \bar{p}= & 4 \sqrt{\gamma} b(\Delta \bar{\lambda}+3 \bar{\lambda})+4 \sqrt{\gamma}\left(D^{C} b\right)\left(D_{C} \bar{\lambda}\right)+\mathcal{L}_{Y} \bar{p} \\
\delta p^{A B}= & \mathcal{L}_{Y} p^{A B}+\sqrt{\gamma}\left(D^{A} D^{B}-\gamma^{A B} \Delta\right) T+\sqrt{\gamma} b\left(\bar{k}^{A B}-D^{A} D^{B} \bar{k}+\gamma^{A B}(\Delta \bar{k}-2 \bar{k})\right) \\
& +\sqrt{\gamma}\left[\left(D^{A} b\right) D_{C} \bar{k}^{C B}+\left(D^{B} b\right) D_{C} \bar{k}^{C A}\right]-2 \sqrt{\gamma}\left[\left(D^{A} b\right)\left(D^{B} \bar{k}\right)+\left(D^{B} b\right)\left(D^{A} \bar{k}\right)\right] \\
& -\sqrt{\gamma}\left(D^{C} b\right)\left(D_{C} \bar{k}^{A B}\right)+3 \sqrt{\gamma} \gamma^{A B}\left(D_{C} b\right)\left(D^{C} \bar{k}\right) \\
\delta p^{r A}= & \mathcal{L}_{Y} p^{r A}+\sqrt{\gamma} D_{B}\left(b \bar{k}^{A B}\right)+\sqrt{\gamma}\left(D^{A} b\right) \bar{k}-\sqrt{\gamma} D^{A} T .
\end{aligned}
$$

\subsubsection{Charges and asymptotic symmetry algebra}

With Henneaux-Troessart parity conditions the charge (2.25) becomes integrable in the functional sense and simplifies to

$$
Q_{M}=\oint d^{2} x\left[4 T \sqrt{\gamma} \bar{\lambda}+W \bar{p}+2 Y_{A}\left(p_{(-2)}^{r A}-2 \bar{\lambda} p^{r A}\right)+2 \sqrt{\gamma} b\left(k^{(2)}-3 \bar{\lambda} \bar{k}\right)\right] .
$$

In contrast to the case of the Regge-Teitelboim parity conditions, the gauge parameters in (2.19)-(2.22) now depend explicitly on the fields through eq. (2.44). Consequently, this field dependence must be taken into account in the computation of the asymptotic 
symmetry algebra. By that reason, the commutator between two surface deformations now contains additional terms that consider the variation of the fields. Thus,

$$
\begin{gathered}
\xi_{3}^{\perp}=\xi_{1}^{i} \partial_{i} \xi_{2}^{\perp}-\xi_{2}^{i} \partial_{i} \xi_{1}^{\perp}+\delta_{2} \xi_{1}^{\perp}-\delta_{1} \xi_{2}^{\perp}, \\
\xi_{3}^{i}=\xi_{1}^{j} \partial_{j} \xi_{2}^{i}-\xi_{2}^{j} \partial_{j} \xi_{1}^{i}+\delta_{2} \xi_{1}^{i}-\delta_{1} \xi_{2}^{i} .
\end{gathered}
$$

The algebra of the asymptotic transformations is then given by

$$
\begin{aligned}
Y_{3}^{A} & =Y_{1}^{B} \partial_{B} Y_{2}^{A}-(1 \leftrightarrow 2), \\
b_{3} & =Y_{1}^{A} D_{A} b_{2}-(1 \leftrightarrow 2), \\
T_{3} & =Y_{1}^{A} D_{A} T_{2}-3 b_{1} W_{2}-\left(D_{A} b_{1}\right)\left(D^{A} W_{2}\right)-b_{1} \Delta W_{2}-(1 \leftrightarrow 2), \\
W_{3} & =Y_{1}^{A} D_{A} W_{2}-(1 \leftrightarrow 2) .
\end{aligned}
$$

It defines an extension of the Carroll algebra with additional symmetry transformations characterized by the even modes with $\ell \geq 2$ of $T(\theta, \phi)$, and by the odd modes with $\ell \geq 3$ of $W(\theta, \phi)$ in the spherical harmonics expansion. The modes with $\ell=0$ of $T$ and $\ell=1$ of $W$ parametrize the symmetry transformations associated with the energy and the linear momentum, respectively.

The canonical generators associated with the above transformations are then given by

$$
\begin{aligned}
\mathcal{T}(\theta, \phi) & =4 \bar{\lambda}, & \mathcal{P}(\theta, \phi) & =\frac{\bar{p}}{\sqrt{\gamma}}, \\
J_{I} & =\oint d^{2} x 2 \sqrt{\gamma} \epsilon_{A B} \hat{r}_{I} D^{A}\left(p_{(-2)}^{r B}-2 \bar{\lambda} p^{r B}\right), & K_{I} & =\oint d^{2} x 2 \sqrt{\gamma} \hat{r}_{I}\left(k^{(2)}-3 \bar{\lambda} \bar{k}\right) .
\end{aligned}
$$

Thus, the charge takes the form

$$
Q_{M}=\vec{\omega} \cdot \vec{J}+\vec{\beta} \cdot \vec{K}+\oint d^{2} x \sqrt{\gamma}[T(\theta, \phi) \mathcal{T}(\theta, \phi)+W(\theta, \phi) \mathcal{P}(\theta, \phi)] .
$$

The generators then have the following non-vanishing Poisson brackets

$$
\begin{array}{rlrl}
\left\{J_{I}, J_{J}\right\} & =-\epsilon_{I J K} J_{K}, & \left\{K_{I}, J_{I}\right\}=-\epsilon_{I J K} K_{K}, \\
\left\{\mathcal{P}(\theta, \phi), J_{I}\right\} & =\hat{Y}_{I}^{A} \partial_{A} \mathcal{P}(\theta, \phi), & \left\{\mathcal{T}(\theta, \phi), J_{I}\right\}=\hat{Y}_{I}^{A} \partial_{A} \mathcal{T}(\theta, \phi), \\
\left\{\mathcal{P}(\theta, \phi), K_{I}\right\} & =\hat{r}_{I}(3 \mathcal{T}+\Delta \mathcal{T})+\left(D_{A} \hat{r}_{I}\right)\left(D^{A} \mathcal{T}\right),
\end{array}
$$

where $\hat{Y}_{I}^{A}:=\frac{\epsilon^{A B}}{\sqrt{\gamma}} \partial_{B} \hat{r}_{I}$. The asymptotic symmetry algebra is canonically realized and corresponds to an extension of the Carroll algebra with additional "Carrollian supertranslations."

\subsection{Example: Magnetic Carrollian Schwarzschild-like solution}

Let us consider the solution of the Carrollian magnetic theory whose spatial metric coincides with the one of the Schwarzschild solution in General Relativity ${ }^{2}$

$$
\begin{array}{lll}
g_{r r}=\frac{1}{\left(1-\frac{M}{8 \pi r}\right)}, & g_{r A}=0, & g_{A B}=r^{2} \gamma_{A B}, \\
\pi^{r r}=0, & \pi^{r A}=0, & \pi^{A B}=0 .
\end{array}
$$

\footnotetext{
${ }^{2}$ The Newton constant was chosen to be $G=\frac{1}{16 \pi}$.
} 
This configuration is spherically symmetric and satisfies the constraints (2.2). In particular, for a vanishing shift, the dynamical equation (2.7) fixes the lapse as

$$
N=\sqrt{1-\frac{M}{8 \pi r}} .
$$

Note that this is exactly the same lapse as in the case of General Relativity, but now there is no notion of a four-dimensional Riemannian metric that can be reconstructed from the spatial metric, the lapse and the shift.

The fields have the following asymptotic expansion:

$$
\begin{aligned}
g_{r r} & =1+\frac{M}{8 \pi r}+\frac{M^{2}}{64 \pi^{2} r^{2}}+\ldots, \\
g_{A B} & =r^{2} \gamma_{A B}, \\
N & =1-\frac{M}{16 \pi r}+\ldots
\end{aligned}
$$

The asymptotic behavior fits within the boundary conditions (2.13)-(2.21) and obeys the Regge-Teitelboim parity condition. Then, using eq. (2.34) the only non-trivial charge is given by the generator

$$
E=2 \oint d^{2} x \sqrt{\gamma} f_{r r}=M
$$

Thus, the energy of this configuration with Regge-Teitelboim parity conditions coincides with the "Schwarzschild mass."

In order to obtain the charges in the case of Henneaux-Troessaert parity conditions, one must perform the following change of coordinates $r \rightarrow r+M /(16 \pi)$. With this choice one has $\bar{k}_{A B}=0$ and

$$
\bar{\lambda}=\frac{M}{16 \pi} .
$$

The only non-trivial generator then corresponds to the zero mode of $\mathcal{T}(\theta, \phi)=4 \bar{\lambda}$ that defines the Carroll energy

$$
E=\oint d \theta d \phi \sin \theta \mathcal{T}=M
$$

\section{Asymptotic symmetries in Electric Carrollian gravity}

\subsection{Action principle, variation of the charge and transformation laws}

The Carrollian theory obtained from the electric contraction of General Relativity has been known for a long time. It can be understood as the "strong coupling limit" of Einstein gravity $(G \rightarrow \infty)$ [38], or alternatively as the limit where the signature of the spacetime vanishes [39]. Its action in canonical form is given by

$$
I=\int d t d^{3} x\left(\pi^{i j} \dot{g}_{i j}-N \mathcal{H}^{E}-N^{i} \mathcal{H}_{i}^{E}\right),
$$

with

$$
\mathcal{H}^{E}=\frac{1}{\sqrt{g}}\left(\pi^{i j} \pi_{i j}-\frac{1}{2} \pi^{2}\right), \quad \mathcal{H}_{i}^{E}=-2 \pi_{i \mid j}^{j}
$$


The constraint $\mathcal{H}^{E}$ is quadratic in the momenta, and does not contain derivatives of the spatial metric. In this sense, the action (3.1) resembles the action of a free relativistic particle, that can be considered as a "gravitational theory in $1+0$ dimensions." This was the starting point of an alternative perturbative approach for quantum gravity [41], where the "free theory" is precisely described by the action (3.1).

The first class constraints obey the same Carrollian surface deformation algebra of the magnetic contraction

$$
\begin{aligned}
& \left\{\mathcal{H}^{E}(x), \mathcal{H}^{E}\left(x^{\prime}\right)\right\}=0, \\
& \left\{\mathcal{H}^{E}(x), \mathcal{H}_{i}^{E}\left(x^{\prime}\right)\right\}=\mathcal{H}^{E}(x) \delta_{, i}\left(x, x^{\prime}\right), \\
& \left\{\mathcal{H}_{i}^{E}(x), \mathcal{H}_{j}^{E}\left(x^{\prime}\right)\right\}=\mathcal{H}_{i}^{E}\left(x^{\prime}\right) \delta_{, j}\left(x, x^{\prime}\right)+\mathcal{H}_{j}^{E}(x) \delta_{, i}\left(x, x^{\prime}\right),
\end{aligned}
$$

and the equations of motion are directly obtained from the action (3.1)

$$
\begin{aligned}
\dot{g}_{i j}= & \frac{2 N}{\sqrt{g}}\left(\pi_{i j}-\frac{1}{2} g_{i j} \pi\right)+N_{i \mid j}+N_{j \mid i}, \\
\dot{\pi}^{i j}= & \frac{N}{2 \sqrt{g}} g^{i j}\left(\pi^{k l} \pi_{k l}-\frac{1}{2} \pi^{2}\right)-\frac{2 N}{\sqrt{g}}\left(\pi_{l}^{i} \pi^{l j}-\frac{1}{2} \pi \pi^{i j}\right) \\
& +\left(N^{k} \pi^{i j}\right)_{\mid k}-N_{\mid k}^{i} \pi^{k j}-N_{\mid k}^{j} \pi^{k i} .
\end{aligned}
$$

In contrast to the magnetic contraction, using eq. (3.6) now it is possible to express the momenta in terms of the time derivatives of the spatial metric.

The canonical generator associated with gauge symmetries takes the form

$$
G\left[\xi, \xi^{i}\right]=\int d^{3} x\left(\xi \mathcal{H}^{E}+\xi^{i} \mathcal{H}_{i}^{E}\right)+Q_{E},
$$

where $\delta Q_{E}$ is given by

$$
\delta Q_{E}=\oint d^{2} s_{l}\left(2 \xi_{k} \delta \pi^{k l}+\left(2 \xi^{k} \pi^{j l}-\xi^{l} \pi^{j k}\right) \delta g_{j k}\right) .
$$

It is worth highlighting that the parameter $\xi$, that characterizes the deformations that move outside the $t=$ const. hypersurfaces does not appear in $\delta Q_{E}$. As it will be discussed in the next sections, this property of the Electric Carrollian theory plays a key role in the form of the asymptotic symmetry algebra, because it does not allow to define a notion of energy.

Under gauge transformations generated by (3.8), the fields $g_{i j}$ and $\pi^{i j}$ transform as

$$
\begin{aligned}
\delta g_{i j}= & \frac{2 \xi}{\sqrt{g}}\left(\pi_{i j}-\frac{1}{2} g_{i j} \pi\right)+\xi_{i \mid j}+\xi_{j \mid i}, \\
\delta \pi^{i j}= & \frac{\xi}{2 \sqrt{g}} g^{i j}\left(\pi^{k l} \pi_{k l}-\frac{1}{2} \pi^{2}\right)-\frac{2 \xi}{\sqrt{g}}\left(\pi_{l}^{i} \pi^{l j}-\frac{1}{2} \pi \pi^{i j}\right) \\
& +\left(\xi^{k} \pi^{i j}\right)_{\mid k}-\xi_{\mid k}^{i} \pi^{k j}-\xi_{\mid k}^{j} \pi^{k i} .
\end{aligned}
$$




\subsection{Asymptotic behavior of the fields}

\subsubsection{Fall-off of the canonical variables}

To describe the asymptotic behavior of the canonical variables, we will consider deviations with respect to the same Carrollian background configuration used in the magnetic theory in eq. (2.12). Since the momenta vanish for this configuration, the constraints of the electric theory in eq. (3.2) are automatically fulfilled. The fall-off of the fields must then guarantee that the symplectic term is finite and that the charges are finite and integrable when appropriate parity conditions are implemented. This is achieved with exactly the same fall-off used in the case of the magnetic contraction in eqs. (2.13)-(2.18), i.e.,

$$
\begin{aligned}
g_{r r} & =1+\frac{f_{r r}}{r}+\frac{f_{r r}^{(-2)}}{r^{2}}+\mathcal{O}\left(r^{-3}\right), \\
g_{r A} & =\frac{f_{r A}^{(-1)}}{r}+\mathcal{O}\left(r^{-2}\right), \\
g_{A B} & =r^{2} \gamma_{A B}+r f_{A B}+f_{A B}^{(0)}+\mathcal{O}\left(r^{-1}\right), \\
\pi^{r r} & =p^{r r}+\mathcal{O}\left(r^{-1}\right) \\
\pi^{r A} & =\frac{p^{r A}}{r}+\frac{p_{(-2)}^{r A}}{r^{2}}+\mathcal{O}\left(r^{-3}\right), \\
\pi^{A B} & =\frac{p^{A B}}{r^{2}}+\mathcal{O}\left(r^{-3}\right) .
\end{aligned}
$$

This asymptotic form is preserved under the transformations (3.10)-(3.11) with gauge parameters of the form

$$
\begin{aligned}
\xi & =r b+f+\mathcal{O}\left(r^{-1}\right), \\
\xi^{r} & =W+\mathcal{O}\left(r^{-1}\right) \\
\xi^{A} & =Y^{A}+\frac{1}{r}\left(\frac{2 b}{\sqrt{\gamma}} p^{r A}+D^{A} W\right)+\mathcal{O}\left(r^{-2}\right),
\end{aligned}
$$

with

$$
Y^{A}=\frac{\epsilon^{A B}}{\sqrt{\gamma}} \partial_{B}(\vec{\omega} \cdot \hat{r}) .
$$

Here, the functions $b=b(\theta, \phi), f=f(\theta, \phi)$ and $W=W(\theta, \phi)$ are general arbitrary scalar functions on the 2 -sphere.

\subsubsection{Variation of the charge}

The conserved charges are obtained by replacing the asymptotic form of the fields in eq. (3.9). There is a naively divergent term of the form

$$
Q_{E}^{\mathrm{div}}=\int d^{2} x 2 Y_{A} p^{r A},
$$

that vanishes provided the order $\mathcal{O}(1)$ of the constraint $\mathcal{H}_{A}^{M}$, given by

$$
p^{r A}+D_{B} p^{A B}=0,
$$


is implemented. Therefore, the charge is finite and integrable in the functional sense. It takes the form:

$$
Q_{E}=\oint d^{2} x\left[2 Y^{A}\left(p^{r r} f_{r A}+p_{A}^{r(-2)}+p^{r B} f_{A B}\right)+\frac{2 b}{\sqrt{\gamma}}\left(p^{r A} p_{A}^{r}\right)+2 W\left(p^{r r}-2 D^{A} p_{r A}\right)\right] .
$$

Note that the parameter $b$ enters in this expression through the order $\mathcal{O}\left(r^{-1}\right)$ of $\xi^{A}$, that is determined by the gauge condition that requires that the order $\mathcal{O}(1)$ of $g_{r A}$ vanishes. As shown later, once parity conditions are imposed, all the terms containing $b$ will no contribute to the charge, and consequently they define pure gauge transformations (proper gauge transformations).

\subsubsection{Transformation laws}

The transformation laws of the fields that are relevant for the parity conditions are given by

$$
\begin{aligned}
\delta f_{r r}= & \mathcal{L}_{Y} f_{r r}+\frac{b}{\sqrt{\gamma}}\left(p^{r r}-\tilde{p}\right), \\
\delta f_{A B}= & \mathcal{L}_{Y} f_{A B}+\frac{2 b}{\sqrt{\gamma}}\left(p_{A B}-\frac{1}{2} \gamma_{A B}\left(\tilde{p}+p^{r r}\right)\right)+2\left(D_{A} D_{B}+\gamma_{A B}\right) W \\
& +\frac{2}{\sqrt{\gamma}}\left(\left(D_{A} b\right) \gamma_{B C} p^{r C}+\left(D_{B} b\right) \gamma_{A C} p^{r C}\right)+\frac{2 b}{\sqrt{\gamma}}\left(\gamma_{B C} D_{A} p^{r C}+\gamma_{A C} D_{B} p^{r C}\right), \\
\delta p^{r r}= & \mathcal{L}_{Y} p^{r r} \\
\delta p^{r A}= & \mathcal{L}_{Y} p^{r A} \\
\delta p^{A B}= & \mathcal{L}_{Y} p^{A B} .
\end{aligned}
$$

Note that the momenta only transform under spatial rotations parametrized by the vector field $Y^{A}$.

\subsubsection{Symplectic term}

The symplectic term takes exactly the same form as in the magnetic case. In particular, the logarithmic divergence takes the form

$$
\int d t d^{3} x \pi^{i j} \dot{g}_{i j} \underset{r \rightarrow \infty}{=} \log (r) \int d t \oint d^{2} x\left(p^{r r} \dot{f}_{r r}+p^{A B} \dot{f}_{A B}\right)+\mathcal{O}\left(r^{-1}\right) .
$$

\subsection{Asymptotic symmetries with Regge-Teitelboim parity conditions}

\subsubsection{Parity conditions}

As in the magnetic case, the Regge-Teitelboim parity conditions defined in eqs. (2.32) and (2.33), render the symplectic term finite by eliminating the logarithmic divergence in (3.28). The consistency of the analysis then requires that the parity conditions are preserved under the transformation laws of the fields (3.23)-(3.27). In particular, from the transformation of $f_{r r}$ in eq. (3.23) one finds that $b(\theta, \phi)$ must be odd. The transformation law of $f_{A B}$ in (3.24) implies that $W(\theta, \phi)$ is even under the antipodal map, with the exception of the mode that is annihilated by the operator $\left(D_{A} D_{B}+\gamma_{A B}\right)$, that corresponds 
to the mode with $\ell=1$ in the spherical harmonics expansion. Therefore, we have the following restrictions on the parameters:

$$
b=b_{\text {odd }}(\theta, \phi), \quad W(\theta, \phi)=\vec{\alpha} \cdot \hat{r}+W_{\text {even }}(\theta, \phi) .
$$

\subsubsection{Charges and asymptotic symmetry algebra}

When Regge-Teitelboim parity conditions are imposed, the charge (3.22) takes the form

$$
Q_{E}=\oint d^{2} x\left[2 \sqrt{\gamma} \vec{\omega} \cdot \hat{r} \epsilon_{A B} D^{A} p_{(-2)}^{r B}+2 \vec{\alpha} \cdot \hat{r}\left(p^{r r}-2 \tilde{p}\right)\right] .
$$

The only parameters that appear in $Q_{E}$ are $\vec{\omega}$ and $\vec{\alpha}$, associated with spatial rotations and spatial translations, respectively. The charge does not depend on the parameters associated with normal deformations, in particular on the parameter describing the time evolution. The origin of this property can be traced back to the absence of spatial derivatives in the constraint $\mathcal{H}^{E}$ in eq. (3.2), which in turn implies that the boundary term associated with the Hamiltonian does not depend on the lapse function, as it can be seen from eq. (3.9).

The asymptotic symmetry algebra can then be obtained using eqs. (2.38) and (2.39) with the following asymptotic form of the parameters

$$
\begin{aligned}
\xi & =\mathcal{O}\left(r^{-1}\right) \\
\xi^{r} & =\vec{\alpha} \cdot \hat{r}+\mathcal{O}\left(r^{-1}\right) \\
\xi^{A} & =\frac{\epsilon^{A B}}{\sqrt{\gamma}} \partial_{B}(\vec{\omega} \cdot \hat{r})+\frac{1}{r} D^{A}(\vec{\alpha} \cdot \hat{r})+\mathcal{O}\left(r^{-2}\right),
\end{aligned}
$$

where only the parameters associated with improper (or large) gauge transformations were included. The algebra then closes according to

$$
\omega_{3}^{K}=-\epsilon_{I J K} \omega_{1}^{I} \omega_{2}^{J}, \quad \alpha_{3}^{K}=-\epsilon_{I J K}\left(\alpha_{1}^{I} \omega_{2}^{J}-\alpha_{2}^{I} \omega_{1}^{J}\right) .
$$

Therefore, the asymptotic symmetry algebra in the electric Carrollian theory with ReggeTeitelboim parity conditions is given by the semi-direct sum of spatial rotations and spatial translations.

The canonical generators then read

$$
J_{I}=2 \oint d^{2} x \sqrt{\gamma} \hat{r}_{I} \epsilon_{A B} D^{A} p_{(-2)}^{r B}, \quad P_{I}=2 \oint d^{2} x \hat{r}_{I}\left(p^{r r}-2 \tilde{p}\right) .
$$

Thus, the charge (3.29) takes the form

$$
Q_{E}=\vec{\omega} \cdot \vec{J}+\vec{\alpha} \cdot \vec{P}
$$

The generators then obey the following algebra in terms of the Poisson brackets

$$
\left\{J_{I}, J_{J}\right\}=-\epsilon_{I J K} J_{K}, \quad\left\{P_{I}, J_{J}\right\}=-\epsilon_{I J K} P_{K}, \quad\left\{P_{I}, P_{J}\right\}=0 .
$$

This is a subalgebra of the Carroll algebra that excludes the generators associated with energy and boosts. 


\subsection{Asymptotic symmetries with Henneaux-Troessaert parity conditions}

\subsubsection{Parity conditions}

The parity conditions introduced by Henneaux and Troessaert [54] can also be implemented in the case of Electric Carrollian gravity. As it was discussed in section 2.4, it is necessary to introduce the new set of variables $\bar{\lambda}, \bar{k}_{A B}, \bar{k}, \bar{p}$ and $k^{(2)}$ defined in eq. (2.40). In terms of these variables, the logarithmic divergence of the symplectic term in eq. (3.28) takes exactly the same form as the one obtained in the case of the magnetic contraction in (2.43). Then, once the parity conditions in (2.41) and (2.42) are imposed, the logarithmic divergence is removed and the symplectic term becomes finite.

In terms of the new variables, the transformation law of the fields in eqs. (3.23)-(3.27) now read

$$
\begin{aligned}
\delta \bar{\lambda}= & \mathcal{L}_{Y} \bar{\lambda}+\frac{b}{4 \sqrt{\gamma}} \bar{p} \\
\delta \bar{k}_{A B}= & \mathcal{L}_{Y} \bar{k}_{A B}+\frac{b}{\sqrt{\gamma}}\left(p_{A B}-\gamma_{A B} \bar{p}\right)+\left(D_{A} D_{B}+\gamma_{A B}\right) W \\
& +\frac{1}{\sqrt{\gamma}}\left(D_{A}\left(b p^{r}{ }_{B}\right)+D_{B}\left(b p^{r}{ }_{A}\right)\right), \\
\delta \bar{p}= & \mathcal{L}_{Y} \bar{p} \\
\delta p^{r A}= & \mathcal{L}_{Y} p^{r A} \\
\delta p^{A B}= & \mathcal{L}_{Y} p^{A B} .
\end{aligned}
$$

The parity conditions of Henneaux and Troessaert must then be preserved under the above gauge transformations. In particular, from the transformation law of $\bar{\lambda}$ in eq. (3.33) one finds that $b(\theta, \phi)$ must be odd under the antipodal map on the 2-sphere. The consistency in the transformation of $\bar{k}_{A B}$ requires that $W$ is also parity odd. Note that $f(\theta, \phi)$ does not appear in these transformations, and consequently it does not have any particular parity. In sum, the parameters $b$ and $W$ obey the following parity conditions

$$
\begin{aligned}
b(\theta, \phi) & (\text { parity odd }) \\
W(\theta, \phi) & \text { (parity odd) }
\end{aligned}
$$

\subsubsection{Charges and asymptotic symmetry algebra}

With Henneaux-Troessart parity conditions, the charge (3.22) simplifies to

$$
Q_{E}=\oint d^{2} x\left[W \bar{p}+2(\vec{\omega} \cdot \hat{r}) \frac{\epsilon^{A B}}{\sqrt{\gamma}} D_{A}\left(p_{(-2)}^{r B}-2 \bar{\lambda} p^{r B}\right)\right]
$$

It depends only on $\vec{\omega}$ and $W(\theta, \phi)$ that correspond to the parameters of spatial rotations and "parity odd supertranslations," respectively. As in the case of Regge-Teitelboim parity conditions, the parameters $b(\theta, \phi)$ and $f(\theta, \phi)$ do not appear in the charges, and therefore they are pure gauge. 
If we consider the asymptotic form of the gauge parameters, where only those associated with non-trivial (improper) symmetries are included, one has

$$
\begin{aligned}
\xi & =\mathcal{O}\left(r^{-1}\right) \\
\xi^{r} & =W+\mathcal{O}\left(r^{-1}\right) \\
\xi^{A} & =\frac{\epsilon^{A B}}{\sqrt{\gamma}} \partial_{B}(\vec{\omega} \cdot \hat{r})+\frac{1}{r} D^{A} W+\mathcal{O}\left(r^{-2}\right) .
\end{aligned}
$$

The asymptotic symmetry algebra can then be obtained using eqs. (2.38) and (2.39). It is characterized by

$$
\begin{aligned}
& \omega_{K}^{3}=-\epsilon_{I J K} \omega_{1}^{I} \omega_{2}^{J}, \\
& W_{3}=Y_{1}^{A} D_{A} W_{2}-Y_{2}^{A} D_{A} W_{1} .
\end{aligned}
$$

This algebra corresponds to the semi-direct sum of spatial rotations with "parity odd supertranslations" parametrized by $\vec{\omega}$ and $W(\theta, \phi)$, respectively. The corresponding canonical generators associated with the above symmetry transformations are then given by

$$
J_{I}=2 \oint d^{2} x \sqrt{\gamma} \epsilon_{A B} \hat{r}_{I} D^{A}\left(p_{(-2)}^{r B}-2 \bar{\lambda} p^{r B}\right), \quad \mathcal{P}(\theta, \phi)=\frac{\bar{p}}{\sqrt{\gamma}},
$$

and are defined in such a way that the charge (3.38) takes the form

$$
Q_{E}=\vec{\omega} \cdot \vec{J}+\oint d^{2} x \sqrt{\gamma} W(\theta, \phi) \mathcal{P}(\theta, \phi) .
$$

They fulfill the following algebra in terms of Poisson brackets:

$$
\left\{J_{I}, J_{J}\right\}=-\epsilon_{I J K} J_{K}, \quad\left\{\mathcal{P}(\theta, \phi), J_{I}\right\}=\hat{Y}_{I}^{A} \partial_{A} \mathcal{P}(\theta, \phi), \quad\left\{\mathcal{P}(\theta, \phi), \mathcal{P}\left(\theta^{\prime}, \phi^{\prime}\right)\right\}=0,
$$

where $\hat{Y}_{I}^{A}:=\frac{\epsilon^{A B}}{\sqrt{\gamma}} \partial_{B} \hat{r}_{I}$. Here $J_{I}$ denotes the generators of spatial rotations, while the odd modes of $\mathcal{P}(\theta, \phi)$ correspond to the "parity odd supertranslations." In particular, the standard spatial translations are given by the modes with $\ell=1$ in the spherical harmonics expansion.

\subsection{Example: Electric Carrollian Schwarzschild-like solution}

In the electric case, one can also find a configuration that solves the constraints (3.2) and the equations of motion (3.6), (3.7) with the same spatial metric components that the Schwarzschild solution in General Relativity.

$$
\begin{array}{lll}
g_{r r}=\frac{1}{\left(1-\frac{M}{8 \pi r}\right)}, & g_{r A}=0, & g_{A B}=r^{2} \gamma_{A B}, \\
\pi^{r r}=0, & \pi^{r A}=0, & \pi^{A B}=0 .
\end{array}
$$

From the dynamical equations (3.6), (3.7), for a spherically symmetric ansatz one finds

$$
N=N(r), \quad N^{A}=0 .
$$


Note that in this case the lapse is not determined by the equations of motion. This is in line with the fact that normal surface deformations do not appear in the charges and that therefore are "pure gauge." The spatial components of the metric then admit the following asymptotic expansion:

$$
\begin{aligned}
g_{r r} & =1+\frac{M}{8 \pi r}+\frac{M^{2}}{64 \pi^{2} r^{2}}+\ldots, \\
g_{A B} & =r^{2} \gamma_{A B} .
\end{aligned}
$$

This behavior fits within the fall-off in eqs. (3.12)-(3.17), and also obeys the ReggeTeitelboim parity conditions. However, in contrast to the magnetic contraction, there are no charges associated with this configuration because $f_{r r}$ does not appear in the charges, and consequently, it can be consistently gauged away.

A similar situation occurs in the case of Henneaux-Troessaert parity conditions, where after performing a radial shift $r \rightarrow r+M /(16 \pi)$, one obtains that $\bar{\lambda}=M /(16 \pi)$. Again, $\bar{\lambda}$ does not appear in the charges, and consequently there are no charges associated with this configuration.

\section{Concluding remarks}

The asymptotic symmetries of the magnetic and electric Carrollian contractions of General Relativity were analyzed in $3+1$ space and time dimensions. The electric gravitational theory was known from long ago as a "strong coupling limit" [38] or as a"zero signature limit" [39] of General Relativity, while the magnetic one was recently introduced by Henneaux and Salgado-Rebolledo in ref. [36] in the context of the study of Carrollian contractions of generic relativistic theories. These Carrollian gravities are described in the Hamiltonian formalism, and the Regge-Teitelboim method was used to determine the asymptotic symmetry algebra, together with their corresponding canonical generators. ${ }^{3}$ In order to render the symplectic term finite, it was necessary to implement appropriate parity conditions on the leading order of the canonical variables, in complete analogy with the case of General Relativity. Two possibilities were explored: Regge-Teitelboim parity conditions [53] and Henneaux-Troessaert parity conditions [54].

In the magnetic theory, for Regge-Teitelboim parity conditions, the asymptotic symmetry algebra corresponds to the finite-dimensional Carroll algebra of Levy-Leblond [1, 2], that can be obtained from an "ultrarelativistic" contraction $(c \rightarrow 0)$ of the Poincaré algebra. On the other hand, when Henneaux-Troessaert parity conditions are imposed, the asymptotic symmetry algebra is given by a "BMS-like" extension of the Carroll algebra by an infinite number of "Carrollian supertranslations." This infinite-dimensional algebra can be recovered from a contraction of the $\mathrm{BMS}_{4}$ algebra written in the non-standard basis used by Henneaux and Troessaert in ref. [54]. It would be interesting to try to make contact with a possible ultrarelativistic contraction of the $\mathrm{BMS}_{4}$ algebra in the standard basis used by Sachs [56], and determine if it can be obtained directly from an asymptotic analysis of

\footnotetext{
${ }^{3}$ The electric gravitational theory was described in a manifestly covariant form under local Carroll symmetries in ref. [40] (see e.g. [36] for a review).
} 
what would be the analog of null infinity in this Carrollian context. A possibility could be the use of the so called "asymptotically null space-like hypersurfaces" introduced in refs. $[57,58]$ in the study of asymptotic symmetries at null infinity in General Relativity using the canonical formalism.

For the theory obtained from the electric contraction, the asymptotic symmetry algebra is truncated to the semi-direct sum of spatial rotations and spatial translations for Regge-Teitelboim parity conditions, and to the semi-direct sum of spatial rotations and an infinite number of parity odd supertranslations in the case of Henneaux-Troessaert parity conditions. In both cases, there are no canonical generators associated with normal displacements to the hypersurfaces defined at constant times in the asymptotic region, that include the time evolution at the boundary. Therefore, these transformations are pure gauge, and consequently there is no notion of energy in this theory. This very special property can be traced back to the absence of spatial derivatives in the Hamiltonian constraint that does not lead to boundary terms associated with it.

These results show a discontinuity between the case of Einstein gravity and its strict electric Carrollian limit. In General Relativity with Regge-Teitelboim parity conditions, the asymptotic symmetry algebra is the ten-dimensional Poincaré algebra [53], even if it is treated perturbatively in the signature parameter [43]. On the other hand, in the strict electric Carrollian limit, the asymptotic symmetries with Regge-Teitelboim parity conditions are described by the six-dimensional algebra spanned by spatial rotations and spatial translations. It possesses less generators than the original one found before the limit. This is particularly interesting because the electric limit was precisely introduced as the zero order in a perturbative expansion in terms of the "signature parameter." This suggests that this jump in the symmetry must be carefully taken into account in this perturbative approach when boundaries are present. A similar situation occurs for Henneaux-Troessaert parity conditions but in terms of the corresponding extended symmetry algebras. On the contrary, the ultrarelativistic limit in the magnetic contraction is perfectly smooth for both parity conditions, at least from the point of view of the asymptotic symmetries. ${ }^{4}$

Different gravitational Carrollian theories were obtained in refs. [48, 49] by considering the gauging of the Carroll algebra. Despite the fact that the relation with the Carrollian gravities obtained from electric and magnetic contractions of General Relativity is not known, in ref. [36] it was conjectured that the action constructed in [48] could be related with the electric contraction, while the action introduced in [49] with the magnetic one. It would be an interesting problem to explore the asymptotic symmetries of the Carrollian gravities of $[48,49]$ because, in principle, they could help to understand the possible relations between the different Carrollian gravitational theories.

The results reported in this article could also be extended to include a negative cosmological constant. As it was pointed out in ref. [36], it is straightforward to modify the

\footnotetext{
${ }^{4}$ In General Relativity there is an additional set of boundary conditions that leads to the BMS algebra at spatial infinity $[59,60]$. The implementation of the analog of these boundary conditions in the Carrollian case was not analyzed in this article. However, Prof. Marc Henneaux pointed out to me that the conclusions are the same as those obtained using Henneaux-Troessaert parity conditions in sections 2.4 and 3.4. I thank Prof. Henneaux for this point.
} 
action principle of the magnetic and electric contractions to incorporate it. However, in contrast to the case with a vanishing cosmological constant, both theories do not share the same Carrollian background configuration. For example, the electric contraction cannot have a background solution with vanishing momenta because this configuration is forbidden by the Hamiltonian constraint. This fact generically leads to striking differences in comparison with the case without a cosmological constant [61].

The generalization to higher dimensions, as well as the coupling to electric/magnetic Carrollian matter fields could also be explored.

\section{Acknowledgments}

I wish to thank Claudio Bunster, Joaquim Gomis, Marc Henneaux, Stefan Prohazka, Ricardo Troncoso and Jakob Salzer for some useful comments. I also thank the hospitality of the Erwin Schrodinger Institute (ESI) in Vienna during the program "Geometry for Higher Spin Gravity: Conformal Structures, PDEs, and Q-manifolds." I am also grateful with Daniel Grumiller for his kind hospitality at the TU-Wien in Vienna, and with Stefan Theisen for his warm hospitality at the Albert Einstein Institute in Golm. This research has been partially supported by Fondecyt grants № 1171162, 1181031, 1181496, 1211226.

\section{A Analysis in Cartesian coordinates with Regge-Teitelboim parity con- ditions}

The results obtained using Regge-Teitelboim parity conditions are reproduced in Cartesian coordinates. This approach resembles the original one introduced by Regge and Teitelboim in General Relativity [53].

Let us consider the same fall-off for the canonical variables as the one used originally by Regge and Teitelboim

$$
\begin{aligned}
& g_{i j}=\delta_{i j}+\frac{h_{i j}^{(1)}(\hat{r})}{r}+\frac{h_{i j}^{(2)}}{r^{2}}+\ldots, \\
& \pi^{i j}=\frac{p_{(2)}^{i j}(\hat{r})}{r^{2}}+\frac{p_{(3)}^{i j}}{r^{3}}+\ldots .
\end{aligned}
$$

In this section the coordinates $x^{i}$, with $i=1,2,3$, denote Cartesian coordinates.

In terms of these variables, the Regge-Teitelboim parity conditions take the form

$$
h_{i j}^{(1)}(\hat{r})=h_{i j}^{(1)}(-\hat{r}), \quad p_{(2)}^{i j}(\hat{r})=-p_{(2)}^{i j}(-\hat{r}) .
$$

The logarithmic divergence of the symplectic is then given by

$$
\int d t d^{3} x \pi^{i j} \dot{g}_{i j} \underset{r \rightarrow \infty}{=} \log (r) \oint d \theta d \phi \sin \theta p_{(2)}^{i j} \dot{h}_{i j}^{(1)}+\ldots
$$

By virtue of the parity conditions (A.3), the divergence is removed and the symplectic term becomes finite. 


\section{A.1 Magnetic Carrollian gravity}

The asymptotic Killing vectors associated with improper gauge transformations can be written as

$$
\xi=\beta_{i} x^{i}+T+\ldots, \quad \xi_{i}=\epsilon_{i j k} x^{j} \omega^{k}+\alpha_{i}+\ldots .
$$

In spherical coordinates these expressions coincide with the ones in eqs. (2.35)-(2.37).

Energy. The energy is obtained for $\xi \underset{r \rightarrow \infty}{\longrightarrow} 1$ and $\xi^{i} \underset{r \rightarrow \infty}{\longrightarrow} 0$ in $\delta Q_{M}$ given by (2.9). Thus

$$
E=\oint d^{2} s_{k}\left(g_{k i \mid i}-g_{i i \mid k}\right)
$$

Note that the expression for the energy in the magnetic Carrollian theory coincides with the formula for the ADM mass in General Relativity [62].

Momentum. The momentum is obtained for $\xi \underset{r \rightarrow \infty}{\longrightarrow} 0$ and $\xi^{i} \underset{r \rightarrow \infty}{\longrightarrow} \alpha^{i}$

$$
Q_{M}[\vec{\alpha}]=2 \alpha_{k} \oint d^{2} s_{i} \pi^{i k}=\alpha_{k} P^{k} .
$$

Angular momentum. The angular momentum is obtained for $\xi \underset{r \rightarrow \infty}{\longrightarrow} 0$ and $\xi_{i} \underset{r \rightarrow \infty}{\longrightarrow}$ $\epsilon_{i j k} x^{j} \omega^{k}$

$$
Q_{M}[\vec{\omega}]=2 \omega^{i} \oint d^{2} s_{l} \epsilon_{i j k} \pi^{l j} x^{k}=\omega^{i} J_{i}
$$

Boosts. Boost generators are obtained when $\xi \underset{r \rightarrow \infty}{\longrightarrow} \beta_{k} x^{k}$ and $\xi^{i} \underset{r \rightarrow \infty}{\longrightarrow} 0$

$$
Q_{M}[\vec{\beta}]=\beta_{k} \oint d^{2} s_{l}\left[x^{k}\left(g_{i l \mid i}-g_{i i \mid l}\right)-g_{k l}+\delta_{k l} g_{i i}\right]=\beta^{k} K_{k} .
$$

\section{A.2 Electric Carrollian gravity}

For the electric contraction, the fall-off of the canonical variables takes exactly the same form as in the magnetic theory, given by eqs. (A.1) and (A.2).

The asymptotic form of the gauge parameters that preserve the above asymptotic conditions is then given by

$$
\begin{aligned}
\xi & =\mathcal{O}\left(r^{-1}\right), \\
\xi_{i} & =\epsilon_{i j k} x^{j} \omega^{k}+\alpha_{i}+\ldots
\end{aligned}
$$

Here, only the terms associated with improper gauge transformations were included.

Momentum. The momentum is obtained when $\xi \underset{r \rightarrow \infty}{\longrightarrow} 0$ and $\xi^{i} \underset{r \rightarrow \infty}{\longrightarrow} \alpha^{i}$

$$
Q_{E}[\vec{\alpha}]=2 \alpha_{k} \oint d^{2} s_{i} \pi^{i k}=\alpha_{k} P^{k} .
$$

Angular momentum. The angular momentum is obtained when $\xi \underset{r \rightarrow \infty}{\longrightarrow} 0$ and $\xi_{i} \underset{r \rightarrow \infty}{\longrightarrow}$ $\epsilon_{i j k} x^{j} \omega^{k}$

$$
Q_{E}[\vec{\omega}]=2 \omega^{k} \oint d^{2} s_{i} \epsilon_{l j k} x^{j} \pi^{i l}=\omega^{k} J_{k}
$$


Open Access. This article is distributed under the terms of the Creative Commons Attribution License (CC-BY 4.0), which permits any use, distribution and reproduction in any medium, provided the original author(s) and source are credited.

\section{References}

[1] J.-M. Lévy-Leblond, Une nouvelle limite non-relativiste du groupe de Poincaré (in French), Ann. I.H.P. Phys. Théor. 3 (1965) 1.

[2] H. Bacry and J. Levy-Leblond, Possible kinematics, J. Math. Phys. 9 (1968) 1605 [inSPIRE].

[3] G. Daŭtcourt, Characteristic hypersurfaces in general relativity. I, J. Math. Phys. 8 (1967) 1492.

[4] R. Fareghbal and A. Naseh, Flat-space energy-momentum tensor from BMS/GCA correspondence, JHEP 03 (2014) 005 [arXiv: 1312.2109] [INSPIRE].

[5] G. Dautcourt, On the ultrarelativistic limit of general relativity, Acta Phys. Polon. B 29 (1998) 1047 [gr-qc/9801093] [INSPIRE].

[6] C. Duval, G.W. Gibbons, P.A. Horvathy and P.M. Zhang, Carroll versus Newton and Galilei: two dual non-Einsteinian concepts of time, Class. Quant. Grav. 31 (2014) 085016 [arXiv: 1402.0657] [INSPIRE].

[7] C. Duval, G.W. Gibbons and P.A. Horvathy, Conformal Carroll groups and BMS symmetry, Class. Quant. Grav. 31 (2014) 092001 [arXiv:1402.5894] [INSPIRE].

[8] E. Bergshoeff, J. Gomis and G. Longhi, Dynamics of Carroll particles, Class. Quant. Grav. 31 (2014) 205009 [arXiv: 1405.2264] [INSPIRE].

[9] R. Fareghbal and A. Naseh, Aspects of flat/CCFT correspondence, Class. Quant. Grav. 32 (2015) 135013 [arXiv: 1408.6932] [inSPIRE].

[10] B. Cardona, J. Gomis and J.M. Pons, Dynamics of Carroll strings, JHEP 07 (2016) 050 [arXiv: 1605.05483] [INSPIRE].

[11] E. Bergshoeff, D. Grumiller, S. Prohazka and J. Rosseel, Three-dimensional spin-3 theories based on general kinematical algebras, JHEP 01 (2017) 114 [arXiv:1612.02277] [INSPIRE].

[12] C. Duval, G.W. Gibbons, P.A. Horvathy and P.M. Zhang, Carroll symmetry of plane gravitational waves, Class. Quant. Grav. 34 (2017) 175003 [arXiv:1702.08284] [INSPIRE].

[13] D. Grumiller, W. Merbis and M. Riegler, Most general flat space boundary conditions in three-dimensional Einstein gravity, Class. Quant. Grav. 34 (2017) 184001 [arXiv: 1704.07419] [INSPIRE].

[14] L. Ciambelli, C. Marteau, A.C. Petkou, P.M. Petropoulos and K. Siampos, Flat holography and Carrollian fluids, JHEP 07 (2018) 165 [arXiv: 1802.06809] [INSPIRE].

[15] J. Figueroa-O'Farrill and S. Prohazka, Spatially isotropic homogeneous spacetimes, JHEP 01 (2019) 229 [arXiv: 1809.01224] [INSPIRE].

[16] A. Barducci, R. Casalbuoni and J. Gomis, Vector SUSY models with Carroll or Galilei invariance, Phys. Rev. D 99 (2019) 045016 [arXiv:1811.12672] [INSPIRE].

[17] K. Morand, Embedding Galilean and Carrollian geometries I. Gravitational waves, J. Math. Phys. 61 (2020) 082502 [arXiv: 1811.12681] [INSPIRE]. 
[18] R.F. Penna, Near-horizon Carroll symmetry and black hole Love numbers, arXiv: 1812.05643 [INSPIRE].

[19] A. Bagchi, A. Mehra and P. Nandi, Field theories with conformal Carrollian symmetry, JHEP 05 (2019) 108 [arXiv: 1901.10147] [INSPIRE].

[20] L. Donnay and C. Marteau, Carrollian physics at the black hole horizon, Class. Quant. Grav. 36 (2019) 165002 [arXiv:1903.09654] [INSPIRE].

[21] J. Figueroa-O'Farrill, R. Grassie and S. Prohazka, Geometry and BMS Lie algebras of spatially isotropic homogeneous spacetimes, JHEP 08 (2019) 119 [arXiv:1905.00034] [INSPIRE].

[22] L. Ravera, AdS Carroll Chern-Simons supergravity in $2+1$ dimensions and its flat limit, Phys. Lett. B 795 (2019) 331 [arXiv:1905.00766] [INSPIRE].

[23] L. Ciambelli, R.G. Leigh, C. Marteau and P.M. Petropoulos, Carroll structures, null geometry and conformal isometries, Phys. Rev. D 100 (2019) 046010 [arXiv:1905.02221] [inSPIRE].

[24] J. Gomis, A. Kleinschmidt, J. Palmkvist and P. Salgado-Rebolledó, Newton-Hooke/Carrollian expansions of (A)dS and Chern-Simons gravity, JHEP 02 (2020) 009 [arXiv: 1912.07564] [INSPIRE].

[25] A. Bagchi, R. Basu, A. Mehra and P. Nandi, Field theories on null manifolds, JHEP 02 (2020) 141 [arXiv: 1912.09388] [inSPIRE].

[26] K. Banerjee, R. Basu, A. Mehra, A. Mohan and A. Sharma, Interacting conformal Carrollian theories: cues from electrodynamics, Phys. Rev. D 103 (2021) 105001 [arXiv: 2008. 02829] [INSPIRE].

[27] D. Grumiller, J. Hartong, S. Prohazka and J. Salzer, Limits of JT gravity, JHEP 02 (2021) 134 [arXiv: 2011.13870] [INSPIRE].

[28] J. Gomis, D. Hidalgo and P. Salgado-Rebolledó, Non-relativistic and Carrollian limits of Jackiw-Teitelboim gravity, JHEP 05 (2021) 162 [arXiv: 2011.15053] [INSPIRE].

[29] A. Bagchi, S. Chakrabortty, D. Grumiller, B. Radhakrishnan, M. Riegler and A. Sinha, Non-Lorentzian chaos and cosmological holography, Phys. Rev. D 104 (2021) L101901 [arXiv: 2106. 07649] [INSPIRE].

[30] P. Concha, D. Peñafiel, L. Ravera and E. Rodríguez, Three-dimensional Maxwellian Carroll gravity theory and the cosmological constant, Phys. Lett. B 823 (2021) 136735 [arXiv:2107.05716] [INSPIRE].

[31] R. Casalbuoni, J. Gomis and D. Hidalgo, World-line description of fractons, arXiv:2107.09010 [INSPIRE].

[32] F. Peña-Benitez, Fractons, symmetric gauge fields and geometry, arXiv:2107.13884 [INSPIRE].

[33] S. Azarnia, R. Fareghbal, A. Naseh and H. Zolfi, Islands in flat-space cosmology, arXiv:2109.04795 [INSPIRE].

[34] A. Campoleoni and S. Pekar, Carrollian and Galilean conformal higher-spin algebras in any dimensions, arXiv:2110.07794 [INSPIRE].

[35] L. Marsot, Planar Carrollean dynamics, and the Carroll quantum equation, arXiv: 2110.08489 [INSPIRE]. 
[36] M. Henneaux and P. Salgado-Rebolledó, Carroll contractions of Lorentz-invariant theories, JHEP 11 (2021) 180 [arXiv:2109.06708] [INSPIRE].

[37] J. de Boer, J. Hartong, N.A. Obers, W. Sybesma and S. Vandoren, Carroll symmetry, dark energy and inflation, arXiv:2110.02319 [INSPIRE].

[38] C.J. Isham, Some quantum field theory aspects of the superspace quantization of general relativity, Proc. Roy. Soc. Lond. A 351 (1976) 209 [inSPIRE].

[39] C. Teitelboim, Surface deformations, their square root and the signature of space-time, in $7^{\text {th }}$ International group theory colloquium: the integrative conference on group theory and mathematical physics, (1978).

[40] M. Henneaux, Geometry of zero signature space-times, Bull. Soc. Math. Belg. 31 (1979) 47.

[41] C. Teitelboim, Quantum mechanics of the gravitational field, Phys. Rev. D 25 (1982) 3159 [INSPIRE].

[42] M. Henneaux, M. Pilati and C. Teitelboim, Explicit solution for the zero signature (strong coupling) limit of the propagation amplitude in quantum gravity, Phys. Lett. B 110 (1982) 123 [INSPIRE].

[43] C. Teitelboim, Quantum mechanics of the gravitational field in asymptotically flat space, Phys. Rev. D 28 (1983) 310 [inSPIRE].

[44] V.A. Belinsky, I.M. Khalatnikov and E.M. Lifshitz, Oscillatory approach to a singular point in the relativistic cosmology, Adv. Phys. 19 (1970) 525 [INSPIRE].

[45] V.a. Belinsky, I.m. Khalatnikov and E.m. Lifshitz, A general solution of the Einstein equations with a time singularity, Adv. Phys. 31 (1982) 639 [INSPIRE].

[46] M. Henneaux, Quantification hamiltonienne du champ de gravitation: une nouvelle approche (in French), Bull. Acad. Roy. Belgique 68 (1982) 940.

[47] T. Damour, M. Henneaux and H. Nicolai, Cosmological billiards, Class. Quant. Grav. 20 (2003) R145 [hep-th/0212256] [INSPIRE].

[48] J. Hartong, Gauging the Carroll algebra and ultra-relativistic gravity, JHEP 08 (2015) 069 [arXiv: 1505.05011] [INSPIRE].

[49] E. Bergshoeff, J. Gomis, B. Rollier, J. Rosseel and T. ter Veldhuis, Carroll versus Galilei gravity, JHEP 03 (2017) 165 [arXiv:1701.06156] [INSPIRE].

[50] C. Teitelboim, How commutators of constraints reflect the space-time structure, Annals Phys. 79 (1973) 542 [INSPIRE].

[51] C. Teitelboim, The Hamiltonian structure of space-time, Ph.D. thesis, unpublished, (1973).

[52] R. Benguria, P. Cordero and C. Teitelboim, Aspects of the Hamiltonian dynamics of interacting gravitational gauge and Higgs fields with applications to spherical symmetry, Nucl. Phys. B 122 (1977) 61 [InSPIRE].

[53] T. Regge and C. Teitelboim, Role of surface integrals in the Hamiltonian formulation of general relativity, Annals Phys. 88 (1974) 286 [INSPIRE].

[54] M. Henneaux and C. Troessaert, BMS group at spatial infinity: the Hamiltonian (ADM) approach, JHEP 03 (2018) 147 [arXiv: 1801.03718] [INSPIRE]. 
[55] H. Bondi, M.G.J. van der Burg and A.W.K. Metzner, Gravitational waves in general relativity. VII. Waves from axisymmetric isolated systems, Proc. Roy. Soc. Lond. A 269 (1962) 21 [INSPIRE].

[56] R. Sachs, Asymptotic symmetries in gravitational theory, Phys. Rev. 128 (1962) 2851 [INSPIRE].

[57] C. Bunster, A. Gomberoff and A. Pérez, Regge-Teitelboim analysis of the symmetries of electromagnetic and gravitational fields on asymptotically null spacelike surfaces, in Tullio Regge: an eclectic genius, from quantum gravity to computer play, L. Castellani, A. Ceresola, R. D'Auria and P. Fré eds., World Scientific, Singapore (2019) [arXiv: 1805.03728] [INSPIRE].

[58] C. Bunster, A. Gomberoff and A. Pérez, Bondi-Metzner-Sachs invariance and electric-magnetic duality, Phys. Rev. D 101 (2020) 044003 [arXiv: 1905.07514] [InSPIRE].

[59] M. Henneaux and C. Troessaert, Hamiltonian structure and asymptotic symmetries of the Einstein-Maxwell system at spatial infinity, JHEP 07 (2018) 171 [arXiv:1805.11288] [INSPIRE].

[60] M. Henneaux and C. Troessaert, The asymptotic structure of gravity at spatial infinity in four spacetime dimensions, arXiv:1904.04495 [INSPIRE].

[61] A. Pérez, Asymptotic symmetries in Carrollian theories of gravity with a negative cosmological constant, CECS-PHY-21/04, work in progress.

[62] R.L. Arnowitt, S. Deser and C.W. Misner, The dynamics of general relativity, Gen. Rel. Grav. 40 (2008) 1997 [gr-qc/0405109] [INSPIRE]. 